\title{
NOISE POLLUTION IN MAIZE MILLING SMES
}

\author{
Erasto Elias*, Bashira A. Majaja*, Said Ibrahim* and Emmanuel G.R. \\ Kizima** \\ *Department of Mechanical and Industrial Engineering, College of \\ Engineering and Technology, University of Dar es Salaam, Tanzania \\ E-mail: ebelias@udsm.ac.tz \\ **Department of Mechanical Engineering, \\ Dar es Salaam Institute of Technology, Tanzania
}

\begin{abstract}
Maize milling SMEs emit high levels of noise which can have adverse effects on human beings and therefore require controlling. This paper reports on a study that involved assessment and measurement of noise level in maize milling SMEs in DSM and Morogoro urban, gathering opinions of workers and the neighbouring population on noise problems, and suggesting solutions for noise control. Noise measurements in 41 SMEs were recorded from 89-103 dBA in DSM and 92-103 dBA in Morogoro near the milling machines. These values were higher than the safe level of $85 \mathrm{dBA}$ for 8 hours working shift. Measurement also indicated high noise in the immediate neighbourhood of the milling plants. Machine operators and neighbours indicated health problems which could be a result of machine noise. Causes of high noise were identified to include loose or untightened machine parts, improper installation of machine system, use of worn out machine bearings, and the use of improperly designed and unbalanced milling hammers. Possible administrative and technical solutions for these problems were suggested. It is concluded from this study that most of maize milling SMEs produce high noise which is indeed pollutant to workers and the neighbouring population. Therefore, it is important to implement the suggested administrative and technical solutions to minimise this problem.
\end{abstract}

Keywords: Maize milling SME, Noise pollution, Noise measurement, Administrative solution, Technical solution.

\section{INTRODUCTION}

\subsection{Background}

Small and Medium Enterprises (SMEs) for maize milling in developing countries such as Tanzania are highly encouraged since they provide income, employment and services to the society. These plants are quite numerous in our society both in rural and urban areas. Most of them provide service to individual customers who need to process flour for domestic consumption. However, over the years there has been an increase of enterprises that process flour for wholesale business instead of waiting for individual domestic customers. The milling process is in most cases accomplished by hammer milling machines, which are electrically or diesel engine driven. Maize milling plants can have adverse effects on the environment and human beings especially when situated in residential places. The plants are characterized by the following problems: (i) High noise, which can be detrimental to the health of milling plant workers and cause disturbances to the neighbouring population; (ii) Flour dust emission which can be detrimental to the health of plant workers who inhale it and the neighbouring surroundings; and (iii) Loss of food in form of emitted flour dust. The level and effects of local maize milling pollution and losses are not known to many stakeholders and therefore are probably neglected or overlooked by SME proprietors, policy makers and other stakeholders out of this ignorance. It therefore calls for investigation to establish basic issues such as: Level of noise and flour dust that subject workers and the neighbouring population; how people are affected by 
the pollution; the amount of flour wasted; and to suggest solutions to improve the situation. In turn, such a study would create awareness to SME proprietors, policy makers and other stakeholders so that appropriate measures can be taken for a better occupational, social and economic situation of the industry.

To understand occupational situation in the maize milling SMEs in Tanzania, preliminary studies were carried out in the then Department of Design and Production Engineering (DPE), University of Dar es Salaam, by Pesambili (2004) and Kizima (2007) with some assistance of Tanzania Gatsby Trust (TGT) funds. In these projects, investigations were done and dust and noise measurements taken in a number of maize milling SMEs in Dar es Salaam. These two studies significantly indicated the existence of pollution problems resulting from the activities of maize milling SMEs. The studies prompted a wider investigation in more areas of the country to confirm the trend and build a case for stakeholders including policy makers to consider. The study was conducted in Dar es Salaam and Morogoro urban under the assistance of Sida/SAREC funds, with the aim of identifying: (i) The sources of dust and noise pollutants in maize milling plants; (ii) The level of noise and dust pollution and its effects to people (plant workers and neighbours) and the environment; (iii) The loss of flour out of the maize milling process; and (iv) Solutions to control noise and dust pollutants in maize milling SMEs.

\subsection{Relevance of the Study to National Policies}

Tanzania Development Vision 2025 (United Republic of Tanzania, 1996a) seeks to transform a low productivity agricultural economy to a semiindustrialized one, led by modernized and highly productive agricultural activities.
For most developing countries, industrial and economic competitiveness hinges on the development of key sectors and issues, particularly those having significant impact towards poverty reduction among people. In Tanzania, such sectors and issues have been identified by the Poverty Reduction Strategy Paper (PRSP) (United Republic of Tanzania, 2001) and they include the agriculture or food security sector, the promotion of the private sector and employment creation.

Agriculture still remains the main source of the household income of the majority of Tanzanians. However, despite the available huge agricultural potential in Tanzania, agro-processing is still at a very low level. One of the major interventions required in the Agricultural Sector for poverty reduction is therefore to develop and promote processing of agriculture based products to take advantage of farm produce with minimum losses. Furthermore, poverty may be reduced through encouraging private sector development and creation of employment particularly among the youth and women groups. It is therefore important to innovate and encourage proper practices in SMEs like maize milling plants, which deal with agroprocessing, so as to enhance the chance of employment to the majority of the people in the society and to ensure food security, in line with the above-mentioned policy documents. More policy documents that advocate innovation; safe and cleaner production practices in manufacturing, agriculture and food processing industries in support of the present study, include the Sustainable Industrial Development Policy (SIDP) (United Republic of Tanzania, 1996b), the National Science and Technology Policy (NSTP) (United Republic of Tanzania, 1996c), the National Environmental Policy (United Republic of Tanzania, 1997), and the National Policy on the Development of 
Small and Medium Enterprises (NPDSME) (United Republic of Tanzania, 2002).

\section{LITERATURE REVIEW}

\subsection{Noise and its Measurement}

Noise can be defined as unwanted or offensive sound that unreasonably intrudes into our daily activities and which may adversely affect the health and well being of individuals. Noise impairs hearing ability, interferes with hearing, causes stress, and hampers concentration and work efficiency (Joshi, 1999). The generation of unreasonable noise within the environment is therefore regarded as a form of pollution because it lowers the quality of life. Noise has many sources, most of which are associated with urban development: road, air and rail transport; industrial noise; neighbourhood and recreational noise.

Although we cannot see sound, it is a force with real dimensions with three definite properties: intensity, frequency and duration. Intensity is the loudness of a sound or the pressure it exerts through the ear. Frequency is the rate at which cycles of high and low sound pressures are produced by a source of sound heard as the pitch of the sound. The human ear hears 20 to $20,000 \mathrm{~Hz}$. Verbal communication is in the range 500 to $3,000 \mathrm{~Hz}$.

The most common instruments used for measuring noise are the sound level meter (SLM), the integrated sound level meter (ISLM) and the noise dosimeter. The SLM consists of a microphone, electronic circuits and a readout display. The microphone detects the small air pressure variations associated with sound and changes them into electrical signals. These signals are then processed by the electronic circuitry of the instrument. The readout displays the sound level in decibels. The SLM takes the sound pressure level at one instant in a particular location. To take measurements, the SLM is held at arm's length at the ear height of those exposed to the noise.

Noise is measured in a unit called "decibel" (dB) which is a measure of how much pressure or sound intensity is created by the sound wave producing the sound as a function of power ratio. An intensity I or power $\mathrm{P}$ is expressed in decibels by the following standard equation:

$I_{\mathrm{dE}}=10 \log _{10}\left(\frac{I}{I}\right)$ or $P_{\mathrm{dE}}=10 \log _{10}\left(\frac{P}{P}\right)$

where $I_{0}$ and $P_{0}$ are a specified reference intensity and power respectively (Martin, 1929). Decibel is a logarithmic scale used to denote the intensity, or pressure level, of a sound relative to the threshold of human hearing. The range of decibels is from 0 to about 140, or from the smallest sound human ears can hear to the sound level that will do immediate and permanent damage to the ear. The noise unit has three scales A, B and C, and the scale closest to human hearing is considered to be the A scale, thus the noise notation "dBA" is used.

\subsection{Typical Noise Levels of Common Systems and Appliances}

The noise levels of many common systems and events measured at the distance that a person would typically be from the source are shown in Table 1. The normal range of hearing begins at approximately " 0 " decibels, but the level at which a person with excellent hearing is able to detect a sound, is in the level of 10 to $15 \mathrm{~dB}$. The other end of the scale is known as the threshold of pain $(140 \mathrm{~dB})$, or the point at which the average person experiences pain.

\subsection{Recommended Noise Intensity Limits}

Sound levels are logarithmic; which means that a small increase in decibels represents a large increase in sound 
energy. For this reason, exposure to sound should be cut in half for every increase of 3 decibels since an increase of $3 \mathrm{~dB}$ represents a doubling of sound power. Similarly, if a signal decreases by $3 \mathrm{~dB}$, half the power is lost. Both the amount of noise and the length of time one is exposed to noise determine its ability to damage one's hearing. The noise standard chart in Table 2 (ACGIH, 1999) gives an indication of average noise intensity limits for everyday sounds around us. An average person can be exposed to a sound source producing $85 \mathrm{dBA}$ for a maximum of eight hours and if it is more than that, protective gears must be used.

\subsection{Effects of Noise}

The health effects of hazardous noise exposure are now considered to be a public health problem. Many research investigations on the effects of noise on human health indicate a variety of health effects. The World Health Organization (WHO) suggests that noise can affect human health and well being in a number of ways such as: to disturb man's work, rest, sleep and communication; it can also damage his/her hearing and evoke other psychological, physiological, and possibly pathological reactions (Australian Environment Council, 1988). Moreover, exposure to noise is also associated with a range of possible physical effects including colds, changes in blood pressure, other cardiovascular changes, increased general medical practice attendance, problems with the digestive system and general fatigue (Job, 1996; U.S. Department of Labour, 1999).

There is fairly consistent evidence that prolonged exposure to noise levels at or above $80 \mathrm{dBA}$ can cause deafness (ISO, 1990). The amount of deafness depends upon the degree of exposure. Since there is strong social pressure to have normal hearing, an individual rarely admits to having a hearing problem until the effects are very substantial. Fortunately, noise hazard can be controlled. No matter what noise problems may be in a particular workplace, measures and methods exist to reduce or control the hazard. Noiseinduced deafness can therefore be prevented and should be prevented. In practice, the effects of noise can be categorized into three major areas:

(a) Physiological effects: noise-induced hearing loss or aural pain, nausea and reduced muscular control, threat to cardiovascular system, systolic blood pressure, and digestive system disorders;

(b) Psychological effects: noise can startle, annoy and disrupt concentration of sleep; and

(c) Interference with communications.

\subsection{NOISE ASSESSMENT IN MAIZE MILLING SMES}

\subsection{Situation Analysis}

Industrial machines and processes produce noise, which can possess a significant occupational health hazard if it is excessive. Maize flour mills present a significant source of excessive noise pollution both on site and in surrounding locality (Evans et al, 2004). Yisa (2005) from his research in food processing industries in Nigeria reported high noise levels ranging from 80 to $110 \mathrm{dBA}$. Studies conducted in a number of milling SMEs in Dar es Salaam by Pesambili (2004) and Kizima (2007) revealed that maize millers are subjected to high levels of noise ranging from 88 to $104 \mathrm{dBA}$, which were above the recommended safe limit of $85 \mathrm{dBA}$ exposure in 8 working hours (Berrekette, 1973). Such high noise, if allowed to be generated, would cause stresses, irritation, headaches and sleeplessness to machine operators and neighbours. This paper examines sources of high noise production and formulation of measures for preventing hearing loss. 
Table 1: Typical sound intensity levels

\begin{tabular}{|c|c|c|}
\hline Common Sounds & $\begin{array}{l}\text { Noise Level } \\
\text { (dB) }\end{array}$ & Effects \\
\hline Jet engine (near) & 140 & Threshold of acute pain \\
\hline $\begin{array}{l}\text { Shotgun firing; Jet takeoff } \\
\text { from ground ( } 30-60 \mathrm{~m} \text { away) }\end{array}$ & 130 & Threshold of pain \\
\hline $\begin{array}{l}\text { Thunderclap (near); } \\
\text { Discotheque }\end{array}$ & 120 & Threshold of sensation \\
\hline $\begin{array}{l}\text { Power saw; Pneumatic drill; } \\
\text { Rock music band }\end{array}$ & 110 & $\begin{array}{l}\text { Regular exposure of more than } 1 \text { minute risks } \\
\text { permanent hearing loss }\end{array}$ \\
\hline Garbage disposal truck & 100 & $\begin{array}{l}\text { Not more than } 15 \text { minutes unprotected exposure } \\
\text { recommended. }\end{array}$ \\
\hline $\begin{array}{l}\text { Subway train; Motorcycle; } \\
\text { Lawnmower }\end{array}$ & 90 & Very annoying \\
\hline $\begin{array}{l}\text { Electric razor; } \\
\text { Many industrial workplaces }\end{array}$ & 85 & $\begin{array}{l}\text { Level at which hearing damage begins } \\
\text { ( } 8 \text { hours exposure) }\end{array}$ \\
\hline Average city traffic noise & 80 & Annoying; interferes with conversation \\
\hline $\begin{array}{l}\text { Vacuum cleaner; Hair dryer; } \\
\text { Inside a car }\end{array}$ & 70 & $\begin{array}{l}\text { Intrusive; interferes with telephone } \\
\text { conversation }\end{array}$ \\
\hline Normal Conversation & 60 & \\
\hline Quiet office air conditioner & 50 & Comfortable \\
\hline Whisper & 30 & Very quiet \\
\hline Normal breathing & 10 & Just audible \\
\hline & 0 & Threshold of normal hearing (1000-4000 Hertz) \\
\hline
\end{tabular}

Source: www.chs.ca/info/noise/levels.html

Table 2: Sound level TLVs for unprotected occupational exposure

\begin{tabular}{cc}
\hline Sound Level (dBA) & Allowable Duration of Exposure (hours) \\
\hline 85 & 8 \\
88 & 4 \\
91 & 2 \\
94 & 1 \\
97 & 0.5 \\
100 & 0.25 \\
Over 103 & No exposure \\
\hline
\end{tabular}

Source: ACGIH (1999)

\subsection{Study Methodology}

The study was undertaken in Dar es Salaam and Morogoro regions. The work concentrated on maize milling plants found in urban areas of these regions, and the study approach adopted included site visits, noise measurements and interviews, and data analysis.

\subsubsection{Site visits to maize milling SMEs} Preliminary study visits were made to maize milling SMEs in Dar es Salaam and Morogoro in order to identify SMEs for detailed investigation. A total of 52 SMEs were randomly visited for this purpose, all of which were easily accessible and willing to take part in the study. This activity involved the following issues:

- Discussing the intention of the project and agreeing with the proprietors and/or plant managers on the subsequent detailed study in the SMEs.

- Assessing the situation in the SMEs and gathering primary information including plant location, apparent level of noise emission, milling equipment or systems in use and nature of business in the SMEs.

\subsubsection{Noise measurements}

Noise measurements were taken using an Integrated Sound Level Meter (ISLM), 
model type VOLTCRAFT DT-8820, with sound measurement range from 35-130 dBA, which is shown in Figure 1. The measurements were taken in 41 SMEs only that were selected from the 52 previsited SMEs.

Along with the measurements, sources of noise in SMEs were to be identified, and all the recorded noise was taken from the milling activities in the plants.

The locations selected at each SME for noise measurement were:

(a) Close to the milling machine (the main noise sources) - at a place where the operator is normally positioned.

(b) Four metres from the milling machine - where other workers and customers would normally be.

(c) Outside the plant - to record possible disturbances to neighbouring population and passers-by.

\subsubsection{Interviewing plant workers and neighbours}

Interviews using questionnaires were conducted using machine operators and the people staying or working in the neighbourhood of the milling plants on a daily basis. The interviews with operators and neighbours were conducted; (i) to gather their views on how they perceived the noise situation, and (ii) obtain qualitatively the kind of health problems they experienced as a result of the noise from the milling plants. A total sampling population of 37 machine operators and 25 neighbours were interviewed. Generally, neighbours were picked for interviewing based on their proximity to a given milling plant and their willingness to participate in the exercise.

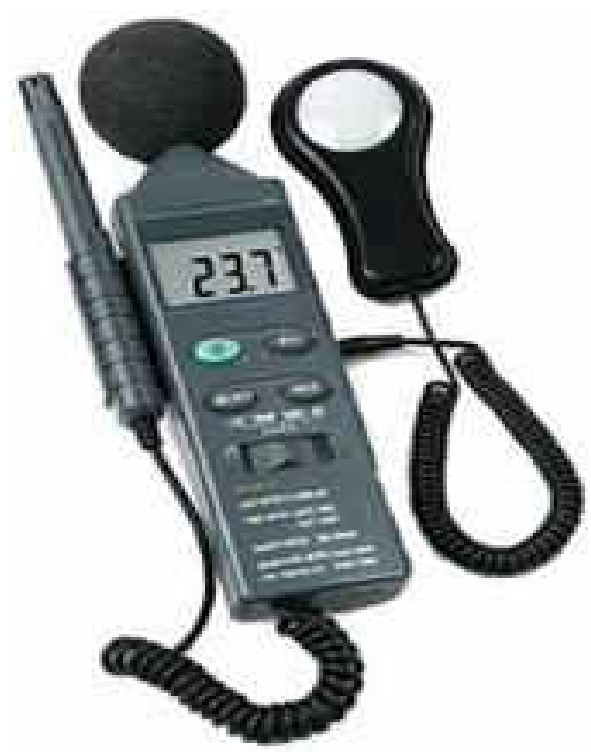

Figure 1: An Integrated Sound Level Meter (ISLM)

\subsubsection{Data analysis}

Noise level data gathered from SMEs that were investigated were tabulated regionwise, analysed, compared and interpreted based on their magnitudes and with standard values. The views of operators and neighbours on various issues and effects of noise were appropriately categorised and presented graphically using Microsoft Excel program.

\section{RESULTS AND DISCUSSION}

\subsection{Noise Level Measurements}

The results of the study involve a total of 27 and 25 maize milling SMEs that were 
visited in Dar es Salaam and Morogoro respectively. All SMEs that were visited were using hammer milling machines with cyclone flour collector. Nearly all machines examined were locally manufactured, except a few in Morogoro which were made in Britain and one in India.

Out of the above visited SMEs, noise levels were recorded in only 23 and 18 maize milling SMEs in Dar es Salaam and Morogoro respectively. The results obtained for milling SMEs in Dar es Salaam and Morogoro are presented in Tables 3 and 4 respectively.

\section{(a) Noise measurements close to the maize milling machine}

The recorded noise level near the machine (i.e. at the operator's working area) in DSM SMEs ranged from 88.8-102.5 dBA while in Morogoro it ranged from 91.9103.2 dBA. Generally, all the investigated SMEs recorded very high noise levels near the machine, where the values were higher than $85.0 \mathrm{dBA}$ which is the recommended limit for 8 hours shift (Berrekette, 1973). The operators who stay close to milling machines on a daily basis for 8 hours or more may not therefore be safe in their hearing ability in the long run.

\section{(b) Noise measurements at four metres} from the maize milling machine

The measurements at this position were meant to assess how other workers (e.g. winnowers) and customers got affected by noise from the machine. In the DSM
SMEs the noise level ranged from 77.693.5 dBA while in Morogoro it ranged from 83.6-99.4 dBA. The other workers and customers are therefore affected by the machine noise albeit at a slightly lower level. They would still feel uncomfortable because the comfortable level for communication, for example, is recommended at $65.0 \mathrm{dBA}$ (Lopez et al., 1992).

\section{(c)Noise measurements carried outside the maize milling plant}

The measurements at this position were meant to indicate how immediate neighbours of milling SMEs and passersby were affected by the noise from the machine. In Dar es Salaam SMEs the noise level ranged from 67.9-85.4 $\mathrm{dBA}$ while in Morogoro it ranged from 70.5-89.8 dBA. Immediate neighbours therefore received a high level of noise although it was not dangerous to their ears. The noise level they received would create an uncomfortable environment as it could make conversation and one's concentration and sleep difficult. For normal conversation, noise in the surroundings has to be lower than $65 \mathrm{dBA}$ (Lopez et al., 1992) while for a good sleep, noise should be lower than $45 \mathrm{dBA}$ (Garcia \& Llopis, 1989; Garcia, 1990).

Figure 2 shows graphical representation of average values of noise levels, as recorded in DSM and Morogoro SMEs. It is indicated that the noise level in the maize milling SMEs was about the same in DSM and Morogoro. 
Table 3: Noise level in maize milling SMEs in Dar es Salaam

\begin{tabular}{cccc}
\hline SME No. & \multicolumn{3}{c}{ Noise Level (dBA) } \\
\cline { 2 - 4 } & $\begin{array}{c}\text { Near milling } \\
\text { machine }\end{array}$ & $\begin{array}{c}\text { 4 m from milling } \\
\text { machine }\end{array}$ & $\begin{array}{c}\text { Outside the } \\
\text { milling plant }\end{array}$ \\
\hline 1 & 88.8 & 83.0 & 72.3 \\
2 & 92.6 & 88.8 & 73.0 \\
3 & 97.0 & 90.4 & 79.7 \\
4 & 92.8 & 84.0 & 72.8 \\
5 & 102.5 & 93.5 & 76.9 \\
6 & 99.3 & 90.2 & 72.6 \\
7 & 95.1 & 87.1 & 77.2 \\
8 & 93.4 & 81.2 & 77.6 \\
9 & 91.9 & 89.6 & 75.2 \\
10 & 90.1 & 77.6 & 67.9 \\
11 & 95.5 & 92.7 & 73.6 \\
12 & 98.2 & 85.6 & 79.1 \\
13 & 93.3 & 85.5 & 81.2 \\
14 & 96.7 & 92.2 & 80.9 \\
15 & 92.2 & 85.2 & 73.0 \\
16 & 97.7 & 87.7 & 85.4 \\
17 & 90.5 & 82.0 & 73.2 \\
18 & 97.7 & 90.5 & 73.8 \\
19 & 95.6 & 84.8 & 72.3 \\
20 & 94.2 & 85.6 & 74.0 \\
21 & 96.8. & 89.4 & 80.0 \\
22 & 92.0 & 85.9 & 80.3 \\
23 & 95.0 & 88.0 & 79.0 \\
\hline Average Values & $\mathbf{9 4 . 7}$ & $\mathbf{8 7 . 0}$ & $\mathbf{7 6 . 0}$ \\
\hline
\end{tabular}

Table 4: Noise level in maize milling SMEs in Morogoro

\begin{tabular}{|c|c|c|c|}
\hline \multirow[t]{2}{*}{ SME No. } & \multicolumn{3}{|c|}{ Noise Level (dBA) } \\
\hline & $\begin{array}{l}\text { Near milling } \\
\text { machine }\end{array}$ & $\begin{array}{l}4 \mathrm{~m} \text { from milling } \\
\text { machine }\end{array}$ & $\begin{array}{l}\text { Outside the } \\
\text { milling plant }\end{array}$ \\
\hline 1 & 91.9 & 83.6 & 70.5 \\
\hline 2 & 103.2 & 99.4 & 89.8 \\
\hline 3 & 103.0 & 94.1 & 80.0 \\
\hline 4 & 100.5 & 94.0 & 84.7 \\
\hline 5 & 94.0 & 87.7 & 74.5 \\
\hline 6 & 92.2 & 85.9 & 71.4 \\
\hline 7 & 98.3 & 89.7 & 74.9 \\
\hline 8 & 96.7 & 88.1 & 77.4 \\
\hline 9 & 98.2 & 89.2 & 83.3 \\
\hline 10 & 97.8 & 88.2 & 79.7 \\
\hline 11 & 94.1 & 92.0 & 80.0 \\
\hline 12 & 96.7 & 87.7 & 75.0 \\
\hline 13 & 92.5 & 88.0 & 76.0 \\
\hline 14 & 96.0 & 86.6 & 78.8 \\
\hline 15 & 98.7 & 92.4 & 82.1 \\
\hline 16 & 102.2 & 94.4 & 86.2 \\
\hline 17 & 99.3 & 88.6 & 81.8 \\
\hline 18 & 93.6 & 86.5 & 77.6 \\
\hline Average Values & 97.2 & 89.8 & 79.1 \\
\hline
\end{tabular}




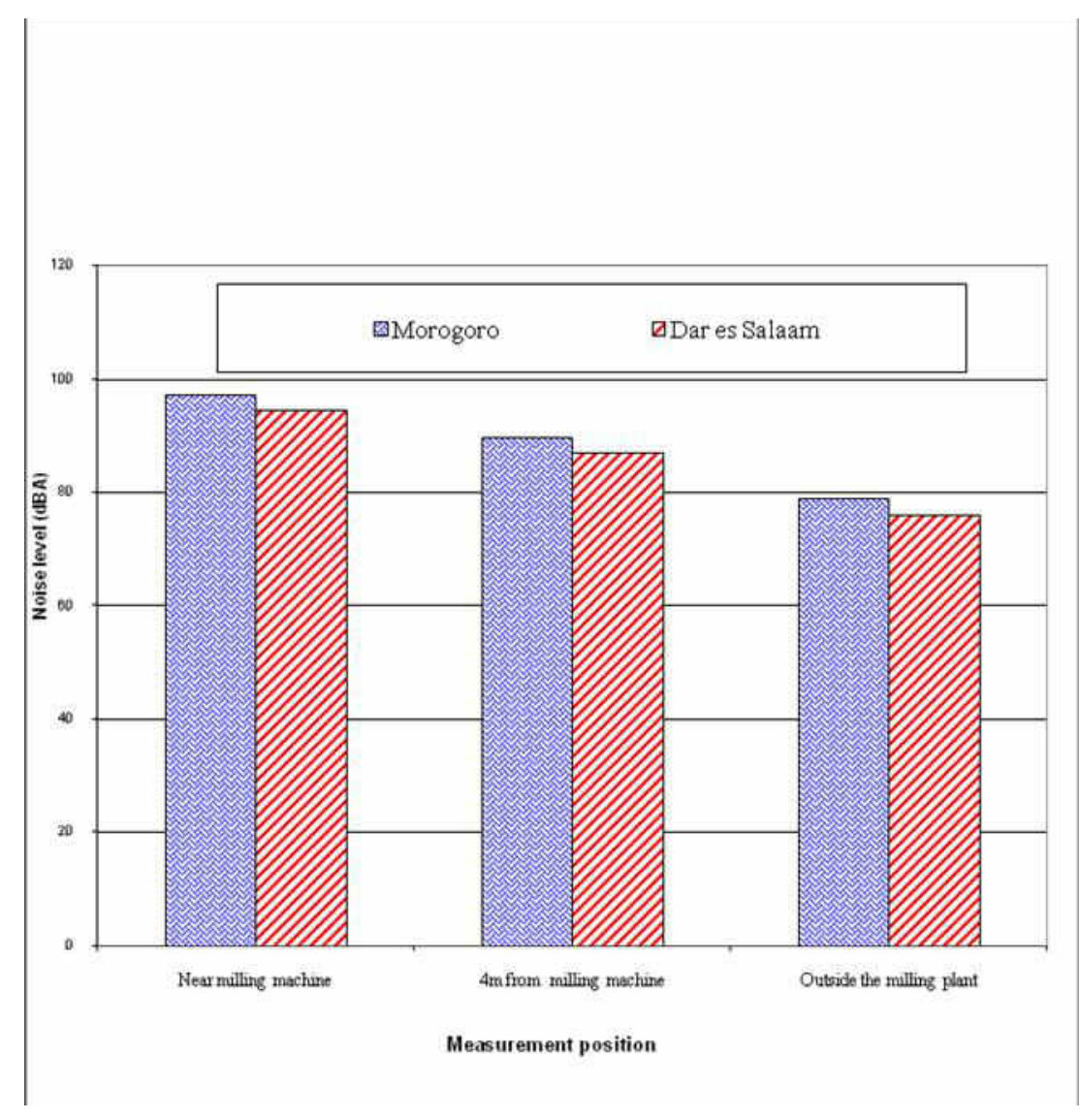

Figure 2: Average noise levels in maize milling SMEs

\subsection{Questionnaire Responses on Machine Noise}

\subsubsection{Operators}

The results obtained from a total of 37 operators that were interviewed on the noise problems, indicated that about $35 \%$ of the respondents admitted having been exposed to a very noisy environment inside the maize milling plants; while other $35 \%$ indicated exposure to a slightly low noisy environment; and the rest could not identify any noise level interference. On the basis of these results, some (about $24 \%$ ) of the respondents reported to have frequently complained to the SME proprietors about noise in the milling plants.

In other findings, 13 respondents did not indicate experiencing any effect of loud noise; but 20 indicated to have been irritated, 3 reported to have headaches and 6 reported that they had stresses. These results are given in Figure 3. Assessment of the problem of communication difficulties in the milling plant while the machine was running showed that about $38 \%$ of the respondents had faced frequent problems, $54 \%$ sometimes faced problems while $8 \%$ did not experience any communication difficulties.

On hearing problems, about $38 \%$ of the respondents admitted to have experienced ringing ears or blurred hearing while $62 \%$ could not confirm having experienced such problems.

\subsubsection{Neighbours}

For the case of 25 neighbours interviewed on machine noise, about $64 \%$ of the 
respondents indicated that they were very much bothered by the noise of neighbouring milling machines, $20 \%$ pointed out to have been less bothered, while $16 \%$ were not at all bothered by the noise. The neighbours' feelings when receiving loud noise were categorised as follows: irritation (19 respondents), headache (9), sleeplessness (7) and normal (6) as presented in Figure 4.

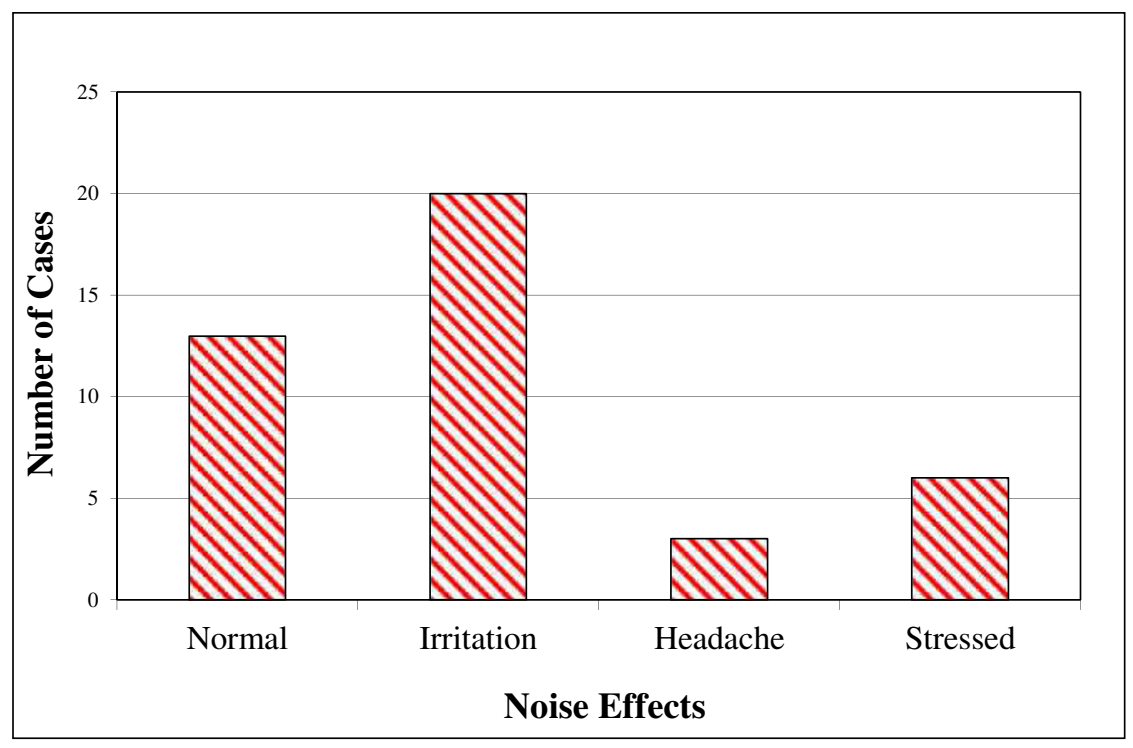

Figure 3: Effects of noise to machine operators in maize milling SMEs

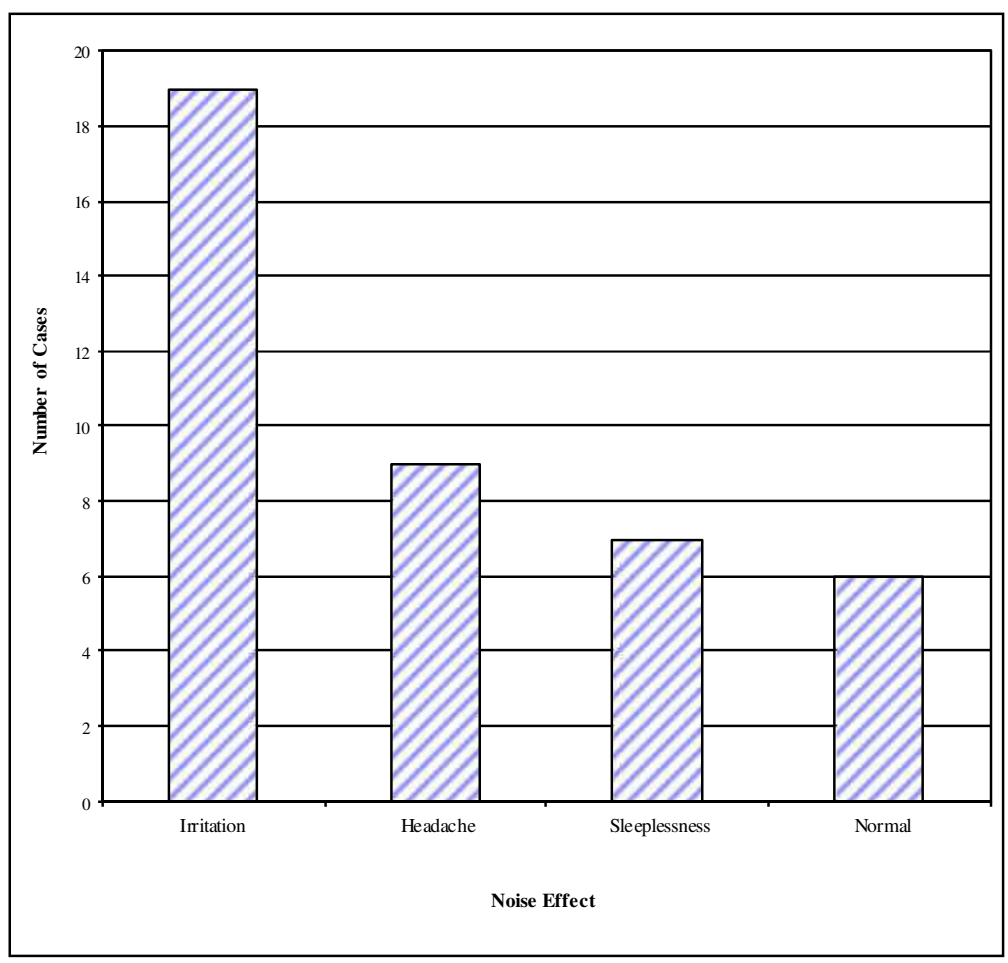

Figure 4: Effects of noise to neighbours of maize milling SMEs 


\section{CONCLUSIONS AND RECOMMENDATIONS}

\subsection{Conclusions}

The results of the study as summarized in this paper indicated that the noise level experienced in all maize milling SMEs exceeded the recommended safe limit of $85 \mathrm{dBA}$ for 8 hours working shift, thus affecting the comfort and health of operators and neighbouring population. It is concluded from this study that most of maize milling SMEs produce high noise which is indeed a pollutant to workers and the neighbouring population. Therefore, it is important to implement administrative and technical solutions to minimise this problem.

\subsection{Recommendations}

The implication of this study is the necessity to keep the levels of noise in maize milling SMEs as low as practicable so as to safeguard the health and convenience of operators and the neighbouring population. In order to achieve this sufficiently, the following are recommended based on the findings of the present study.

\subsubsection{Administrative solutions}

(a)Developing and practicing repair culture: Milling SMEs need to practice repair culture especially in dealing with the following issues which are related to noise production.

(i) Fixing loose machine parts;

(ii) Replacing defective bearings; and

(iii) Replacing worn out machine hammers.

(b)Employing enough operators for shift activities and shift rotation.

(c) Use of PPEs such as ear plugs and ear muffs against noise.

\subsubsection{Technical solutions}

(a) Proper installation of milling machines: sufficient fixation and application of vibration damping materials.

(b) Properly designed foundation for machine and motor installation to minimize machine vibration and therefore noise level. It was observed during the study that most milling machines (especially in Dar es Salaam) were installed on ordinary floors meant for domestic or light business buildings.

(c) Carrying out a study to improve design and material specification of milling hammers, followed by standardization by TBS so that manufacturers can use standard hammers.

(d) Establishing proper balancing procedure of milling hammers.

(e) Use of proper thickness $(2 \mathrm{~mm})$ of materials for milling machine hopper and cyclone.

\section{ACKNOWLEDGEMENT}

This research was conducted with financial support from Sida/SAREC through the University of Dar es Salaam Research Support Fund. We therefore wish to express our sincere gratitude to Sida/SAREC and the then Directorate of Research and Publication of the University of Dar es Salaam for this financial support.

\section{REFERENCES}

ACGIH (1999). Threshold Limit Values for Chemical Substances and Physical Agents and Biological Exposure Indices, ACGIH, Cincinnati, OH, USA.

Australian Environment Council (1988). Community Response to Noise in Australia, Results of the National Noise Survey, Report No. 21, AGPS, Canberra Citations: 123 . https://www.environment.nsw.gov.au 
/soe/97/ch1/16.htm. [Accessed on 5/1/2008].

Barrekette S.E. (1973). Pollution Engineering and Scientific solutions. Plenum Press, New York - London, 572-573.

Evans J.P., Whyte R.T., Price J.S., Bacon, J. M., Semple D. A. and Scarlett A.J. (2004). Practical Solutions to Noise Problems in Agriculture. Silsoe Research Institute, Wrest Park, Silsoe, Bedford, MK45 4HS.

Garcia A. and Llopis G.A. (1989). Alteraciones del Suenó producida ruidal ambiental. Gaceta sanitaria, 12(3): 421-426.

Garcia A. (1990). Community response to environmental noise at Valencia. Environment International Report, 16: 533-541.

International Organization for Standardization (ISO) (1990). Determination of Occupational Noise Exposure and Estimation of Noiseinduced Hearing Impairment, Acoustics $2^{\text {nd }}$ Edition, Geneva, Switzerland.

Job R.F.S. (1996). The Influence of Subjective Reactions to Noise on Health Effects of the Noise, Environmental International, 22(1): 93-104.

Joshi S.J. (1999). Air Pollution Control in Cement Industry, Indian Cement Review, June.

Kizima E.G.R. (2007). Control of Noise and Dust Pollution from Maize Milling Machines. MSc. Dissertation, UDSM.

Lopez B.I., Carles J.L. and Herranz K. (1992). The effect of noise abatement programme on the attention capacity and reading ability of school. $14^{\text {th }}$ International Congress on Acoustics, Beijing China.

Martin W.H. (1929). DeciBel: the New Name for the Transmission Unit, Bell System Technical Journal, January.
Pesambili L.C. (2004). A study towards developing systems to reduce noise and dust pollution from maize milling machines. MSc. Dissertation, UDSM.

The Canadian Hearing Society, Noise/Sound Levels and Human Response.

http:/www.chs.ca/info/noise/levels.ht $\underline{\mathrm{ml}}$ [Accessed 18/11/2007].

United Republic of Tanzania, Vice President's Office, (1996a). Tanzania Development Vision 2025, Dar es Salaam.

United Republic of Tanzania, Ministry of Industries and Trade (1996b). Sustainable Industrial Development Policy, Dar es Salaam.

United Republic of Tanzania, Ministry of Science, Technology and Higher Education, (1996c). The National Science and Technology Policy, Dar es Salaam.

United Republic of Tanzania, Vice President's Office (1997). National Environmental Policy, Dar es Salaam.

United Republic of Tanzania, Vice President's Office, 2001. Poverty Reduction Strategy, Dar es Salaam.

United Republic of Tanzania, Ministry of Industries and Trade, 2002. National Policy for the Development of Small and Medium Enterprises, Dar es Salaam.

U.S. Department of Labour - OSHA, (1999). OSHA's Rationale for the noise exposure PEL; Washington DC. www.osha.gov/pls/oshaweb/ [Accessed 27/10/2007].

Yisa M.G. (2005). African Newsletter on Occupational Health and Safety; 15(7$10)$. http://www.ttl.fi/AfricanNewsletter [Accessed 27/10/2007]. 